\title{
Expanded progenitor populations, vitreo-retinal abnormalities, and Müller glial reactivity in the zebrafish leprechaun/patched2 retina Jonathan Bibliowicz ${ }^{1,2}$ and Jeffrey M Gross*1,2,3
}

\author{
Address: ${ }^{1}$ Section of Molecular Cell and Developmental Biology, The University of Texas at Austin, Austin, TX, USA, ${ }^{2}$ Institute of Cell and \\ Molecular Biology, The University of Texas at Austin, Austin, TX, USA and 3Institute for Neuroscience, The University of Texas at Austin, Austin, \\ TX, USA \\ Email: Jonathan Bibliowicz - yoni@mail.utexas.edu; Jeffrey M Gross* - jmgross@mail.utexas.edu \\ * Corresponding author
}

Published: 19 October 2009

BMC Developmental Biology 2009, 9:52 doi:10.1/86/147|-2/3X-9-52
Received: I May 2009

Accepted: 19 October 2009

This article is available from: http://www.biomedcentral.com/l47/-2/3X/9/52

(C) 2009 Bibliowicz and Gross; licensee BioMed Central Ltd.

This is an Open Access article distributed under the terms of the Creative Commons Attribution License (http://creativecommons.org/licenses/by/2.0), which permits unrestricted use, distribution, and reproduction in any medium, provided the original work is properly cited.

\begin{abstract}
Background: The roles of the Hedgehog $(\mathrm{Hh})$ pathway in controlling vertebrate retinal development have been studied extensively; however, species- and context-dependent findings have provided differing conclusions. Hh signaling has been shown to control both population size and cell cycle kinetics of proliferating retinal progenitors, and to modulate differentiation within the retina by regulating the timing of cell cycle exit. While cell cycle exit has in turn been shown to control cell fate decisions within the retina, a direct role for the Hh pathway in retinal cell fate decisions has yet to be established in vivo.
\end{abstract}

Results: To gain further insight into Hh pathway function in the retina, we have analyzed retinal development in leprechaun/patched2 mutant zebrafish. While lep/ptc2 mutants possessed more cells in their retinas, all cell types, except for Müller glia, were present at identical ratios as those observed in wild-type siblings. lep/ptc2 mutants possessed a localized upregulation of GFAP, a marker for 'reactive' glia, as well as morphological abnormalities at the vitreo-retinal interface, where Müller glial endfeet terminate. In addition, analysis of the over-proliferation phenotype at the ciliary marginal zone (CMZ) revealed that the number of proliferating progenitors, but not the rate of proliferation, was increased in lep/ptc2 mutants.

Conclusion: Our results indicate that Patched2-dependent Hh signaling does not likely play an integral role in neuronal cell fate decisions in the zebrafish retina. ptc2 deficiency in zebrafish results in defects at the vitreo-retinal interface and Müller glial reactivity. These phenotypes are similar to the ocular abnormalities observed in human patients suffering from Basal Cell Naevus Syndrome (BCNS), a disorder that has been linked to mutations in the human PTCH gene (the orthologue of the zebrafish ptc2), and point to the utility of the lep/ptc2 mutant line as a model for the study of BCNS-related ocular pathologies. Our findings regarding CMZ progenitor proliferation suggest that, in the zebrafish retina, Hh pathway activity may not affect cell cycle kinetics; rather, it likely regulates the size of the retinal progenitor pool in the CMZ. 


\section{Background}

During retinal development, proliferation and differentiation must be tightly coordinated in order to produce a tissue of the proper size and containing the correct cell types [1]. The Hh pathway has been shown to play a critical role in controlling these two seemingly opposite processes [2]. Early in retinal development, the optic vesicle is composed of a population of proliferating neuroepithelial cells that will ultimately give rise to the mature retina [3]. Differentiation of the retinal neuroepithelium occurs in a succession of temporally overlapping waves [4]. In the zebrafish, the first cells to exit the cell cycle become retinal ganglion cells (RGCs), which differentiate in a Sonic Hh (Shh)-dependent wave [5]. A second Hhdependent wave of differentiation in the inner nuclear layer (INL) occurs almost simultaneously with the first wave, and is responsible for the differentiation of the multiple cell types of the INL (horizontal, amacrine, and bipolar cells, and Müller glia) and the rod and cone photoreceptors of the outer nuclear layer (ONL) [6]. In addition, extra-retinal Hh signaling originating in the retinal pigment epithelium (RPE) has been suggested to direct photoreceptor differentiation [7]. While the role of the Hh pathway in cell cycle exit and differentiation of retinal progenitors is well described, comparatively less is known about its possible influence on cell fate decisions. In Xenopus, over-expression of p27Xic1, which promotes cell cycle exit, results in increased numbers of early-born cell types (RGCs), while the over-expression of cyclin E1, which inhibits cell cycle exit, biases cell fate towards lateborn cell types (e.g. Müller glia) [8]. Similarly, Shh has been shown to promote early cell cycle exit in the Xenopus retina [9]; however, a direct role of the Hh pathway in dictating retinal cell fate has yet to be established in vivo.

While the cells of the central retina of the zebrafish exit the cell cycle by 60 hours post fertilization (hpf) [10], a population of retinal progenitors at the $\mathrm{CMZ}$ is maintained and continues to proliferate throughout the animal's lifetime $[11,12]$. The spatial pattern of cells within the $\mathrm{CMZ}$, with retinal stem cells at the most peripheral region followed by proliferative retinal progenitors and finally differentiating progenitors more centrally, is thought to recapitulate the temporal sequence of early retinal development [13]. Some of the factors that control early retinal development, such as notch, $r \times 1$, pax $6 a$, and $v s \times 2 / \operatorname{ch} x 10$, are expressed in the zebrafish CMZ [11]. In Xenopus, expression of gli2, gli3, and smoothened, is found at the retinal margin, suggesting a role for the Hh pathway in the stem cell/progenitor population in the CMZ [14]. Shh overexpression studies in chick support a role for the Hh pathway as a mitogen in the CMZ [15], consistent with its described mitogenic effects on retinal progenitors in early zebrafish and mouse retinal development [16,17].
Invaluable knowledge regarding Hh function in the developing retina has been gained from the analysis of $\mathrm{Hh}$ pathway mutants in zebrafish. For example, the zebrafish mutants syu (shh) and smu (smoothened) have helped elucidate the complex mechanisms of Hh-dependent neural differentiation and proliferation $[7,16]$. However, retinal differentiation is severely defective or altogether absent in these mutants due to defective Hh signaling, making it difficult to arrive at definitive conclusions about the possible role of the Hh pathway in cell fate decisions. To address this issue, we analyzed retinal development in the zebrafish lep/ptc2 mutant, in which the Hh pathway is 'over-active'. lep/ptc2 mutants possess a non-sense mutation in the exon encoding the sixth trans-membrane domain of Patched2 [18], which is predicted to abolish its function $[18,19]$. Loss of Patched function results in an 'over-active' Hh pathway due de-repression of Smoothened [20]. Normally, in the absence of Hh ligand, the Patched protein inhibits the activity of Smoothened. Binding of the Hh ligand to its Patched receptor relieves this inhibition, ultimately resulting in increased transcription of Hh target genes $[21,22]$. A non-functional Patched would therefore result in loss of inhibition on, and constitutive activity of, Smoothened in ptc2 deficient cells. lep/ ptc2 mutants possess increased proliferation in multiple tissues including the retinal $\mathrm{CMZ}$ [18]. Utilizing this mutant as a tool, we sought to gain further insight into $\mathrm{Hh}$ function with respect to retinal cell fate decisions and to determine how Hh activity influences progenitor proliferation at the CMZ. Our results revealed no significant change in neuronal composition within the retina, but we did note a reduction in differentiated Müller glia that was accompanied by localized Müller glial reactivity and abnormalities at the vitreo-retinal interface. In addition, the results of cell cycle analyses suggest that the over-proliferation at the $\mathrm{CMZ}$ likely results from an expansion of the progenitor cell population, and not from direct effects of the mutation on cell cycle kinetics within progenitor cells.

\section{Results}

lep/ptc2 mutants possess enlarged retinas and overgrown irises, while lens and RPE morphology are largely normal

lep/ptc2 mutants were isolated based on expanded PCNA staining in multiple tissues including the retinal $\mathrm{CMZ}$ [18]. While mutant embryos appear to be of normal size at 5 days post fertilization (dpf) (Figure 1A), their retinas are enlarged (Figure 1D, E) and they possess reduced pupil size ([18] and Figure 1B, C). To characterize ocular tissues in lep/ptc2 mutants, we performed histological analyses at $33 \mathrm{hpf}$, around the time of the onset of retinal differentiation, and at 5dpf, when the laminar organization of the embryonic retina is complete $[10,23]$. lep/ptc2 retinas appear largely normal at $33 \mathrm{dpf}$ and do not possess increases in retinal cell number relative to phenotypically 

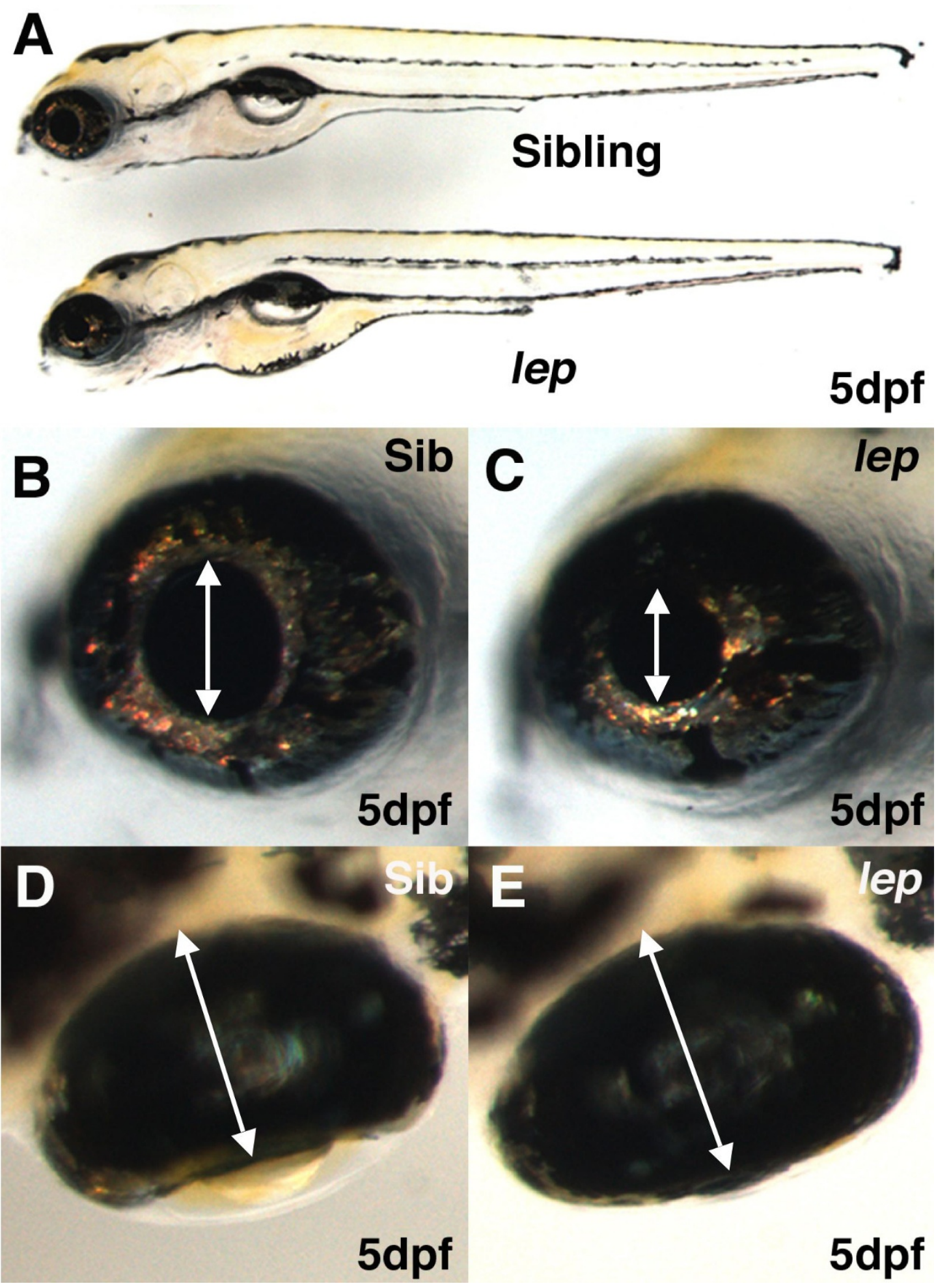

Figure I

lep/ptc2 mutants possess enlarged retinas and reduced pupil size. Whole embryo lateral view shows that lep/ptc2 embryos are largely normal compared to their siblings (A). High magnification images of sibling (B) and lep/ptc2 (C) eyes reveal reduced pupil size in lep/ptc2 (arrows in B, C). Dorsal view images illustrate the overgrowth of retinal tissue as the retina is thicker and appears to engulf the lens (arrows in D, E). 
wild-type siblings (Figure 2A, B, E). At 5dpf, however, lep/ $p t c 2$ retinal cells are more tightly packed and mutant retinas contain a larger number of cells as compared to their phenotypically wild-type siblings (Figure 2C, D, E). Normal retinal lamination of differentiated neurons has been shown to be dependent on Shh signaling in vitro [24]. Lamination is largely normal in lep/ptc2 mutants and all major cell types are present (Figure 2C, D). Loss-of-function experiments in both frog and mouse have suggested a role for the Hh pathway in the development of the RPE $[14,25]$. In lep/ptc2 mutants the RPE is of normal thickness and morphology. The Hh pathway has also been implicated in lens development [26,27], and the reduced pupil size present in lep/ptc2 mutants was previously attributed to potential degeneration of the lens [28]. No gross morphological defects are evident in the lenses of lep/ptc2 mutants at either 33hpf or 5dpf, however. Additionally, histological analyses revealed an abnormality in the anterior segment of lep/ptc2 eyes where the iris was over-grown and extended further over the lens than in sibling retinas (Figure 2D). The enlarged iris and the overgrowth of the $\mathrm{CMZ}$ are the likely causes of the decreased pupil size, rather than defects in lens morphology.

\section{Neuronal composition of the lep/ptc2 retina is normal, while Müller glia are under-represented}

Hh signaling has been shown to be required for the differentiation of multiple cell types in the vertebrate retina $[6,7,29]$ and is thought to promote cell fate decisions by modulating the timing of cell cycle exit [30]. Shh gain-offunction experiments in Xenopus link a faster cell cycle with early cell cycle exit [9], which in turn results in an
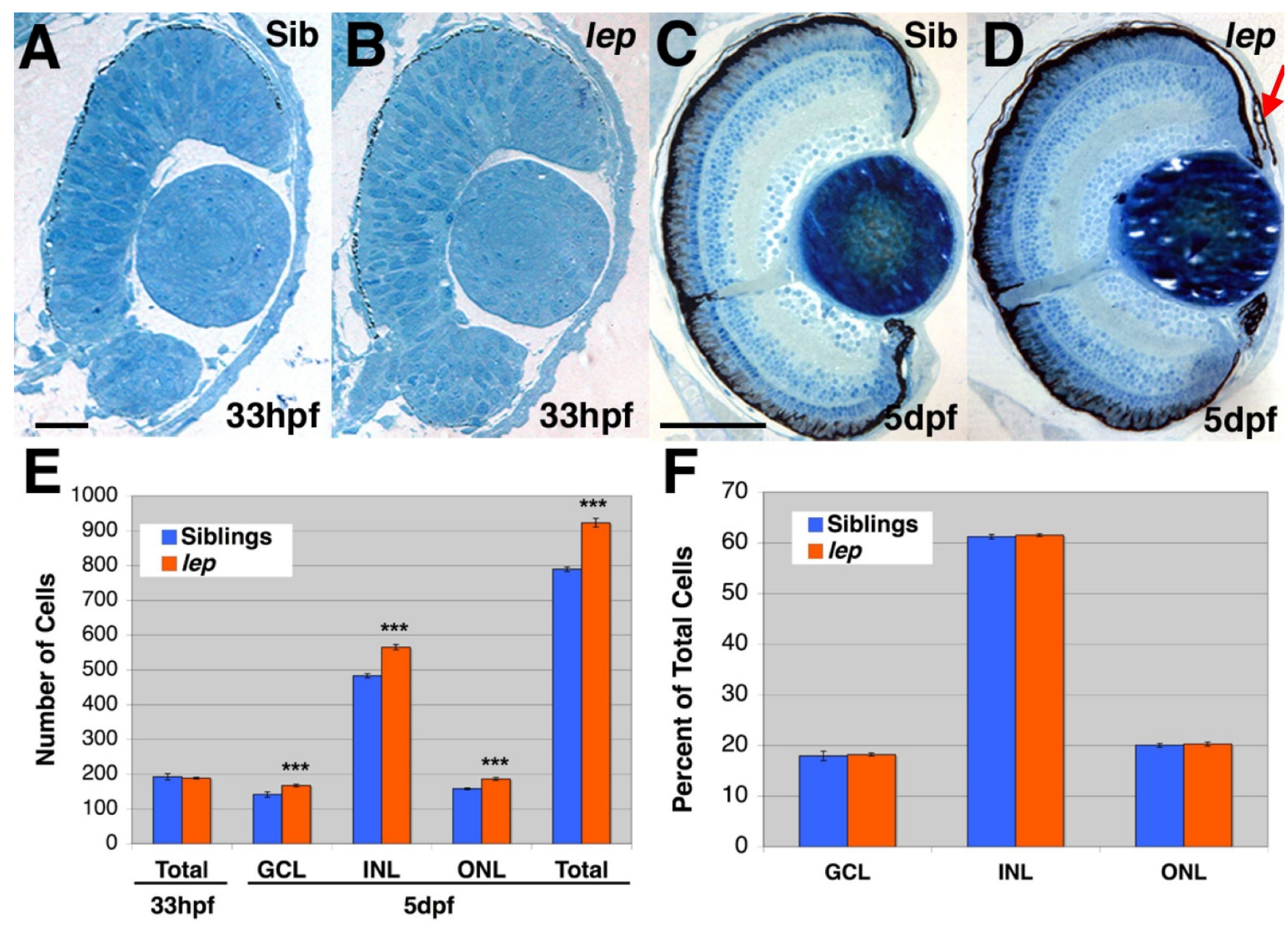

Figure 2

lep/ptc2 mutant retinas possess proportional increases in cell numbers in all three nuclear layers. Histology of sibling $(A, C)$ and lep/ptc2 mutant (B, D) retinas at $33 \mathrm{hpf}(A, B)$ and $5 \mathrm{dpf}(C, D)$. E) Quantification of total number of retinal cells at $33 \mathrm{hpf}$ and $5 \mathrm{dpf}$, and of cells in each nuclear layer at $5 \mathrm{dpf}(18.0 \%, 17.0 \%$, and $17.8 \%$ increases in GCL, INL, and ONL, respectively. $\left.{ }^{* * *} \mathrm{p}<0.000 \mathrm{I}\right)$. F) Graph of the proportion of each nuclear layer as a percentage of total retinal cells at $5 \mathrm{dpf}$. Scale Bars $=20$ um in $A, B$ and 100 um in C, D. 
over-production of early-born retinal cell types at the expense of late-born cell types [8]. We therefore reasoned that in lep/ptc2 retinas, where the Hh pathway is overactive, early-born cell types would be over-represented while late-born cell types would be reduced in number. Cell counts from histological sections of lep/ptc2 mutants revealed a statistically significant increase in cell number in all three retinal nuclear layers (GCL, INL, and ONL), as well as in total retinal cells, when compared to those from phenotypically wild-type siblings (Figure 2E). However, no significant change in the proportion of each layer was observed when the number of cells per layer was calculated as a percentage of total retinal cells (Figure 2F). This suggests that all three retinal cell layers are proportionally increased in $l e p / p t c 2$ retinas.

It is possible that in lep/ptc2 mutants, an overactive Hh pathway could alter neuronal composition within each layer. To test this hypothesis, immunohistochemistry using markers for each specific cell type in the INL and ONL was performed on retinal sections at $5 \mathrm{dpf}$, when all cell types are normally differentiated and the embryonic retina is fully developed. Since the GCL layer is almost exclusively composed of retinal ganglion cells (RGCs), no further analysis was performed on this layer. In order to analyze cell types within the INL, immunohistochemistry was performed using antibodies that mark amacrine cells (5E11), bipolar cells (PKC) and Müller glia (Glutamine Synthetase, GS), while horizontal cells were detected according to their stereotypical morphology in histological sections. This analysis revealed that while the proportion of each neuronal cell type in the INL was not significantly changed in $l e p / p t c 2$ mutants, there was a statistically significant decrease in the proportion of differentiated Müller glia (Figure 3). For the $\mathrm{ONL}$, immunohistochemistry was performed to detect rods (Zpr3) and each three cone opsins (blue, red/green, and UV) [31]. Due to the diffuse nature of the staining of some of the markers used, precise cell counts were not performed on these cell types. However, immunohistochemisty did show that these cell types are present in lep/ptc2 and qualitatively appear unaffected (Figure 4). In all, lep/ ptc2 mutants possess more cells in their retinas and each cell type, except for Müller glia, was present at identical ratios to those observed in phenotypically wild-type siblings.

\section{lep/ptc2 mutants possess abnormalities at the vitreo- retinal boundary and localized Müller glial reactivity}

Müller glial endfeet terminate at, and contribute to, the inner limiting membrane (ILM), which separates the retina from the vitreous [32]. Interestingly, our immunohistochemical analysis of differentiated Müller glia revealed disruptions in the integrity of the ILM (Figure 5A, B). Since humans who possess mutations in the PTCH gene (orthologue of the zebrafish ptc2 [33]) have been previously shown to possess abnormalities in the vitreo-retinal interface [34], we sought to further investigate this phenotype. High magnification examination of GS-stained retinas revealed disruptions in the integrity of the ILM in $40 \%$ of the lep/ptc2 retinas examined. While Müller glial endfeet form a tight and continuous boundary at the vitreoretinal boundary in sibling retinas, the ILM in lep/ptc2 retinas was often discontinuous and disorganized, and endfeet did not appear to properly terminate in the ILM (Figure 5A, B). In Ptc1+/- mice, ocular abnormalities are accompanied by the upregulation of GFAP, a marker of reactive Müller glia, and ectopic proliferation of Müller glia in the central retina [34].

Immunohistochemical analysis of GFAP revealed that its levels in Müller glia were indeed higher in lep/ptc2 mutant retinas as compared to their phenotypically wild-type siblings (Figure 5C, C', D, D'). Higher levels of GFAP were often localized to Müller glial endfeet in the central retina, adjacent to the optic nerve. However, BrdU incorporation assays in $l e p / p t c 2$ mutants at $5 \mathrm{dpf}$ did not reveal ectopic proliferation in the central retina (see Additional file 1), suggesting that the upregulation of GFAP is not coupled with Müller glial proliferation in this context. In addition, the localized nature of Müller glial reactivity in not due to abnormal cell death in the lep/ptc2 retina and/or optic nerve since no apoptotic nuclei were detected by TUNEL assays (see Additional file 1).

\section{patched2 (ptc2) is expressed in the progenitor/stem cell populations of the $\mathrm{CMZ}$}

We next sought to better understand the nature of the over-proliferation at the CMZ by defining the retinal cell populations that express the ptc2 transcript. At 56hpf, prior to the time that the overgrowth phenotype becomes detectable, ptc2 expression was visible at the retinal margin, corresponding to the undifferentiated population of retinal progenitors of the CMZ (Figure 6A). Expression continued to be restricted to the $\mathrm{CMZ}$ at $72 \mathrm{hpf}$ (Figure 6B), consistent with previously reported expression of $\mathrm{Hh}$ target genes in the Xenopus CMZ [14]. Closer inspection of $p t c 2$ expression in the $\mathrm{CMZ}$ at $72 \mathrm{hpf}$ revealed that lowlevel ptc2 expression was detected throughout most of the $\mathrm{CMZ}$, consistent with previous reports [35]. Staining was more robust in two distinct CMZ cell populations; the first was immediately apposed to the RPE and the second was a patch of cells at the edge of the CMZ, bordering the differentiated GCL (arrows in Figure 6E). Hh ligands have been shown to be expressed in both the RPE [14] and the GCL $[5,24]$ during retinal development, and Patched genes are expressed in a Hh-dependent manner [33]. Thus, these CMZ regions may reflect areas of active Hh signaling. Finally, the ptc2 transcript is expressed at the 

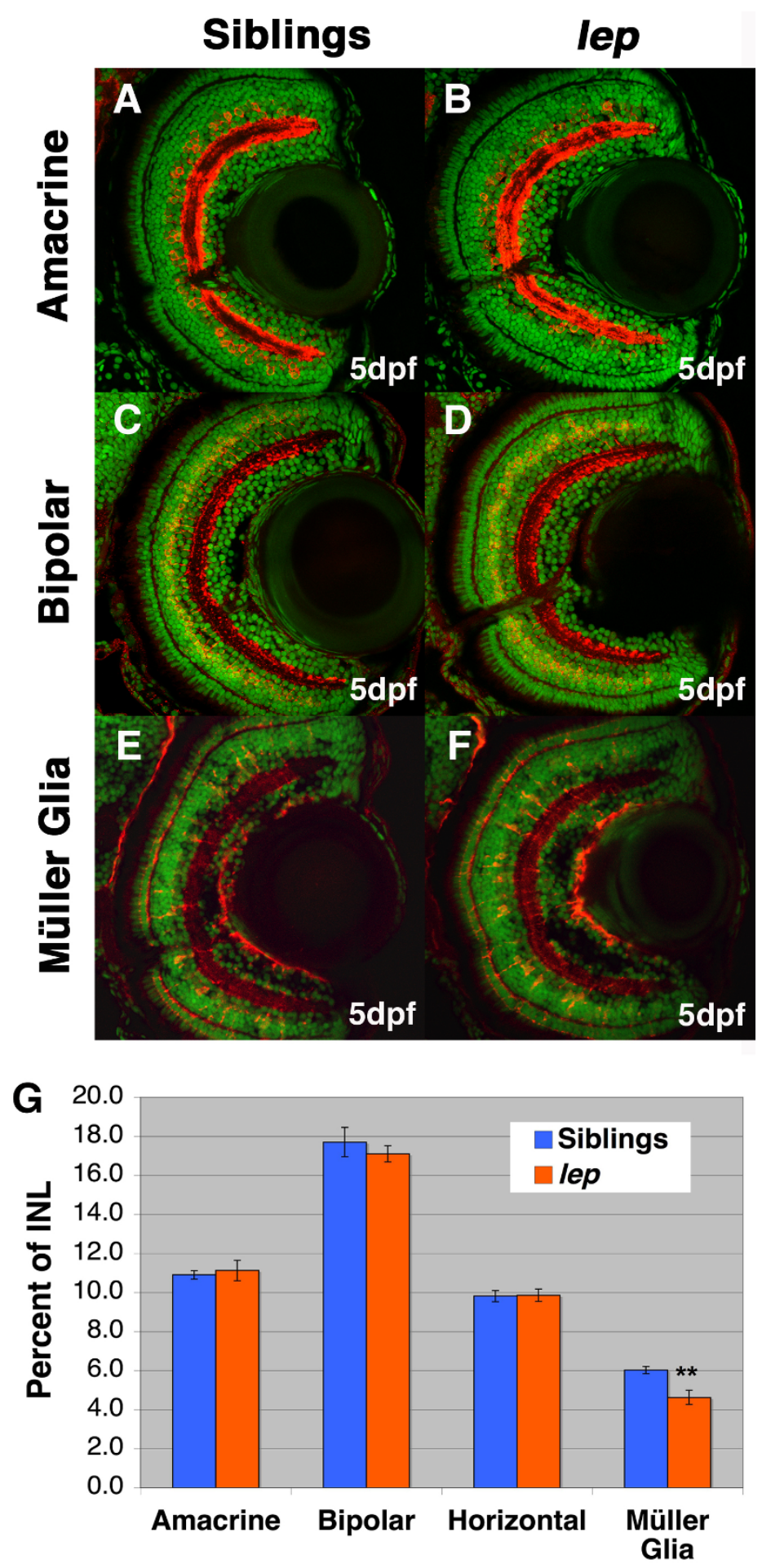

Figure 3

All neuronal cell types in the lep/ptc2 INL are increased proportionately, while Müller glia are reduced. Immunohistochemistry of sibling $(A, C, E)$ and lep/ptc2 (B, D, F) retinas for Amacrine cells $(A, B)$, Bipolar cells $(C, D)$, and Müller glia (E, F). G) Cell counts for each INL cell type. No significant change is cell number in all neuronal cell types when calculated as a percentage of total INL cells. Müller glia, however, were reduced in lep/ptc2 retinas by $29 \%$ relative to sibling retinas $\left(*^{*} \mathrm{p}=\right.$ $0.0013)$. 

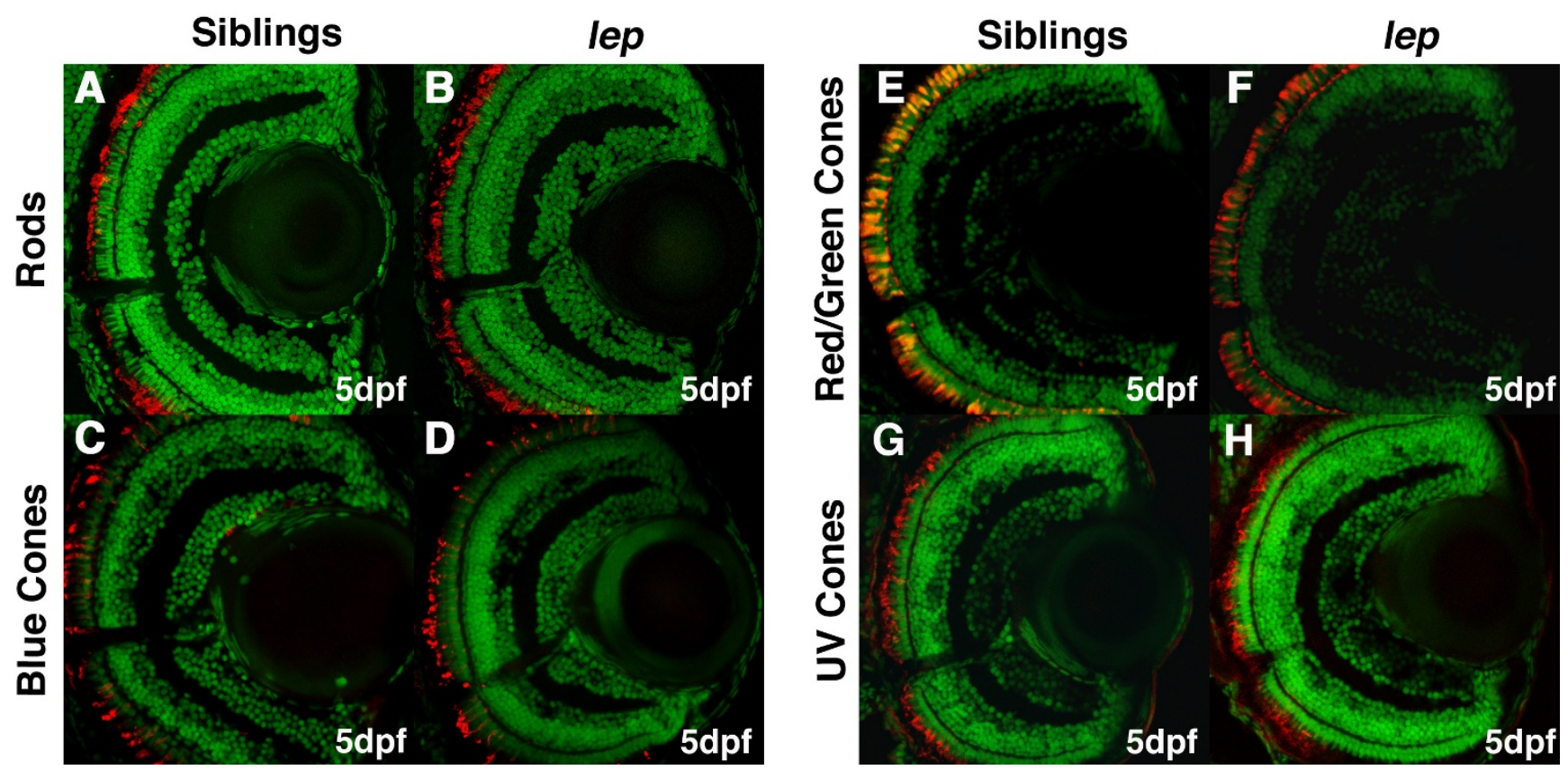

Figure 4

Photoreceptors appear unaffected in lep/ptc2 retinas. Immunohistochemical analysis of rods (A, B), blue cones (C, D), red/green cones $(E, F)$, and UV cones $(G, I)$ in sibling $(A, C, E, G)$ and lep/ptc2 retinas $(B, D, F, I)$. All photoreceptor types were present and appear normal.

iridio-corneal angle, where the iris and cornea converge [36] (arrows in Figure 6B).

\section{The number of proliferating progenitors, but not the rate of progenitor proliferation, is increased in the lep/ptc2 \\ CMZ}

Ptc1 $1^{+/-}$mice possess increased numbers of progenitor cells at all stages of retinal development, suggesting a role for the Hh pathway in controlling the size of retinal progenitor populations in the ciliary margin, and these mice also possess a persistent progenitor population in the retinal margin as adults [37]. Studies in post-hatch chicks suggest that Shh regulates the proliferation of $\mathrm{CMZ}$ progenitors [15]. In addition, Shh over-expression studies in Xenopus have revealed a role for the Hh pathway in regulating the length of the cell cycle in the progenitor populations of the CMZ [9]. Given the differing roles for Hh pathway activity in these contexts, we wanted to determine whether the over-proliferation in lep/ptc2 retinas was due to an increase in the number of proliferating cells and/or an increase in the proliferation rate of the progenitor cell population in the CMZ. To address these possibilities, we first performed a 'pulse-chase' BrdU analysis on lep/ptc2 and sibling embryos starting at $64 \mathrm{hpf}$, when a mild retinal phenotype is first detectable. Embryos were sorted according to phenotype and exposed to a short pulse (15 min) of BrdU. Half of the embryos were immediately fixed for sectioning, while the other half were transferred to nonBrdU containing fish water and fixed 8 hours later (72hpf). This enabled us to determine the rate of progenitor proliferation in both lep/ptc2 and sibling retinas during the 8 hour chase period. The results of these assays revealed that, on average, lep/ptc2 retinal sections contained $67 \%$ more BrdU-positive cells in the CMZs when compared to phenotypically wild-type sibling retinas at 64hpf (Figure 7A, B, E). At 72hpf, after the 8 hour 'chase', the average number of BrdU-positive cells in lep/ptc2 and phenotypically wild-type sibling retinal sections increased by almost identical ratios (2.29-fold changes in lep/ptc2 vs. 2.31 fold change in sibling sections, Figure 7C, D, E). From these data, we can therefore infer that, on average, each proliferating cell gave rise to 2.3 daughter cells during the 8 hour 'chase' period. To further analyze proliferation, we assayed the expression of phosphohistone $\mathrm{H} 3$ (PH3) and these analyses revealed that the proportion of $\mathrm{CMZ}$ progenitors occupying the $\mathrm{M}$ phase of the cell cycle increased by approximately $34 \%$ in lep/ptc2 retinas at $64 \mathrm{hpf}$, when compared to their phenotypically wild-type siblings (Figure 7E, F, H). The degree of this increase was almost identical to that observed in progenitor cells occupying the $\mathrm{S}$ phase using BrdU as a marker (Figure 7A, B, $H)$, suggesting that the length of the $S$ and $M$ phases was not changed relative to the other. These results support a model in which the expanded CMZ of lep/ptc2 mutants 


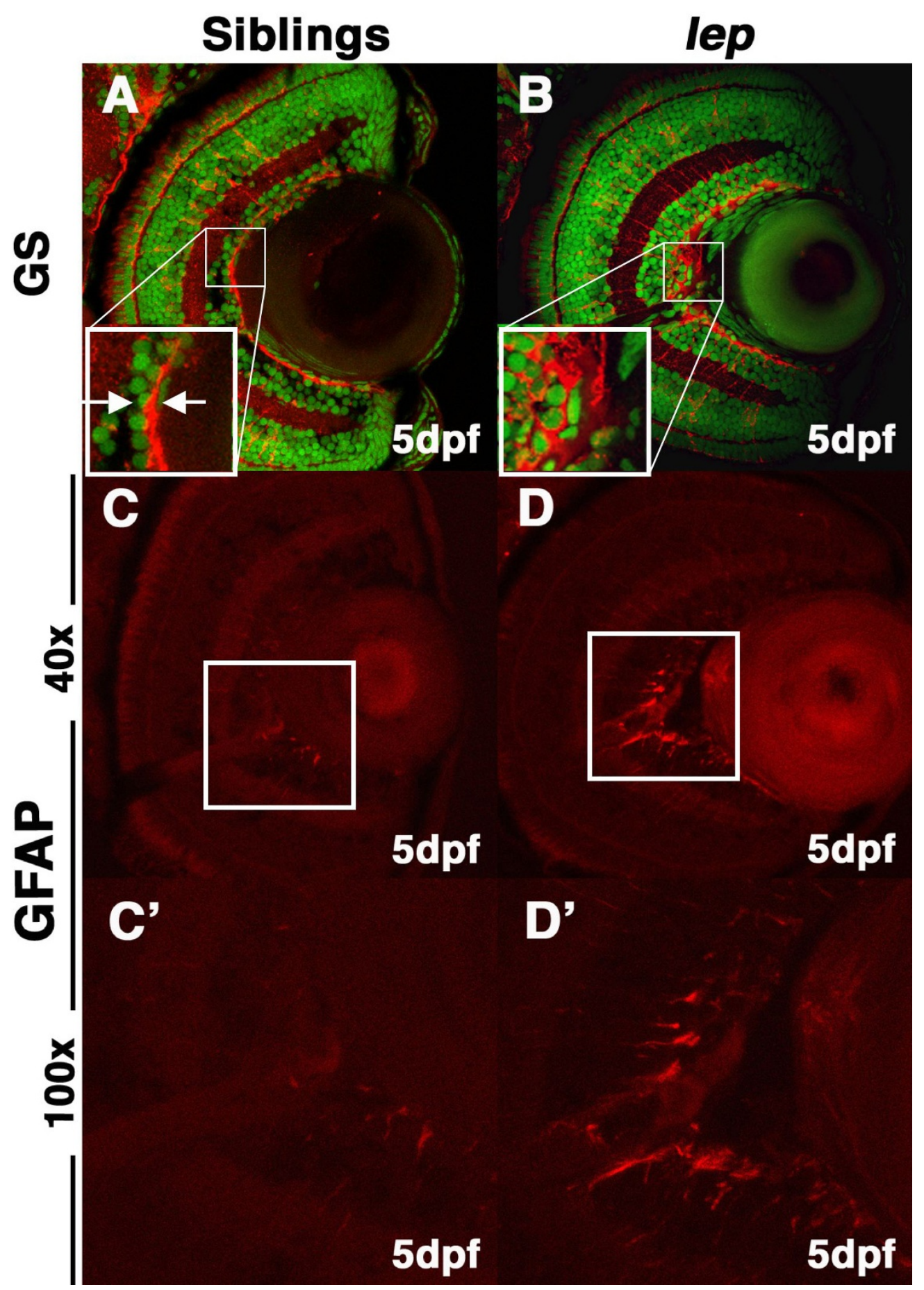

\section{Figure 5}

lep/ptc2 mutants display Müller glial reactivity and morphological abnormalities in the ILM. Immunohistochemical analysis using glutamine synthetase (GS) antibody, which marks differentiated Müller glia and their endfeet at the ILM, highlights disruptions in the ILM. (A) In sibling retinas, the ILM is tight and continuous (inset). (B) In lep/ptc2 retinas the ILM is discontinuous and Müller glial endfeet do not terminate properly at the ILM (inset). Glial fibrillary acidic protein (GFAP) antibody staining reveals elevated immuno-reactivity in the inner retina, adjacent to the optic nerve of lep/ptc2 (D and arrows in D') mutant retinas as compared to siblings (C, $\left.C^{\prime}\right)$. Approximately $40 \%$ of analyzed mutants displayed significant ILM disruptions and elevated GFAP levels. 


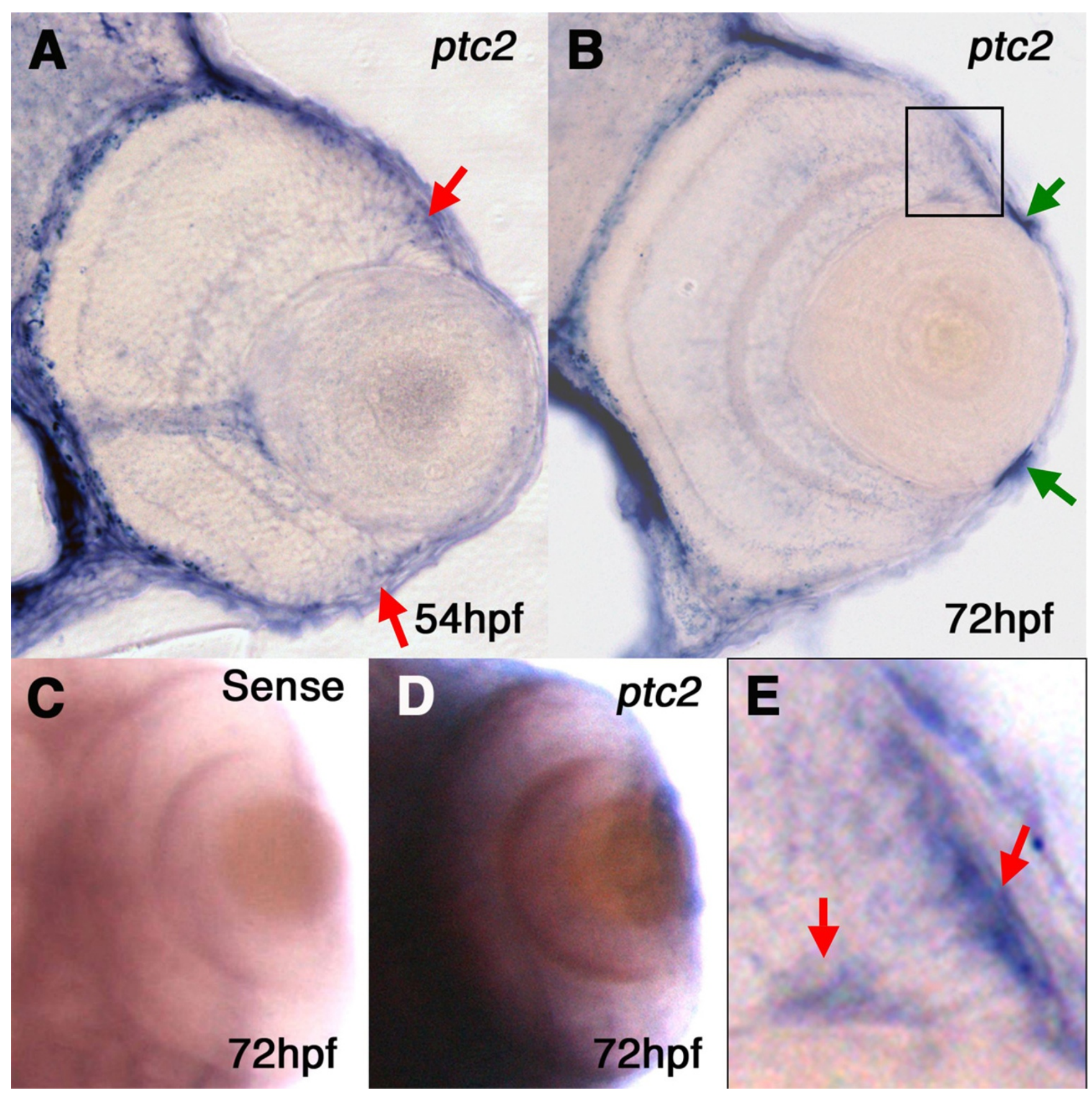

\section{Figure 6}

ptc2 is expressed at the zebrafish $\mathbf{C M Z}$. In-situ hybridization for the ptc2 transcript shows expression at the retinal margin at $56 \mathrm{hpf}$ (arrows in A). At $72 \mathrm{hpf}$, transcript is detected throughout the $\mathrm{CMZ}$ (B) and at the iridio-corneal angle (green arrows), with high magnification of CMZ region $(E)$ revealing more robust staining adjacent to the RPE and in a region just apposed to the GCL (red arrows). Whole mount images from a dorsal view of sense (C) and anti-sense (D) probes in-situ hybridization show that staining is specific. 

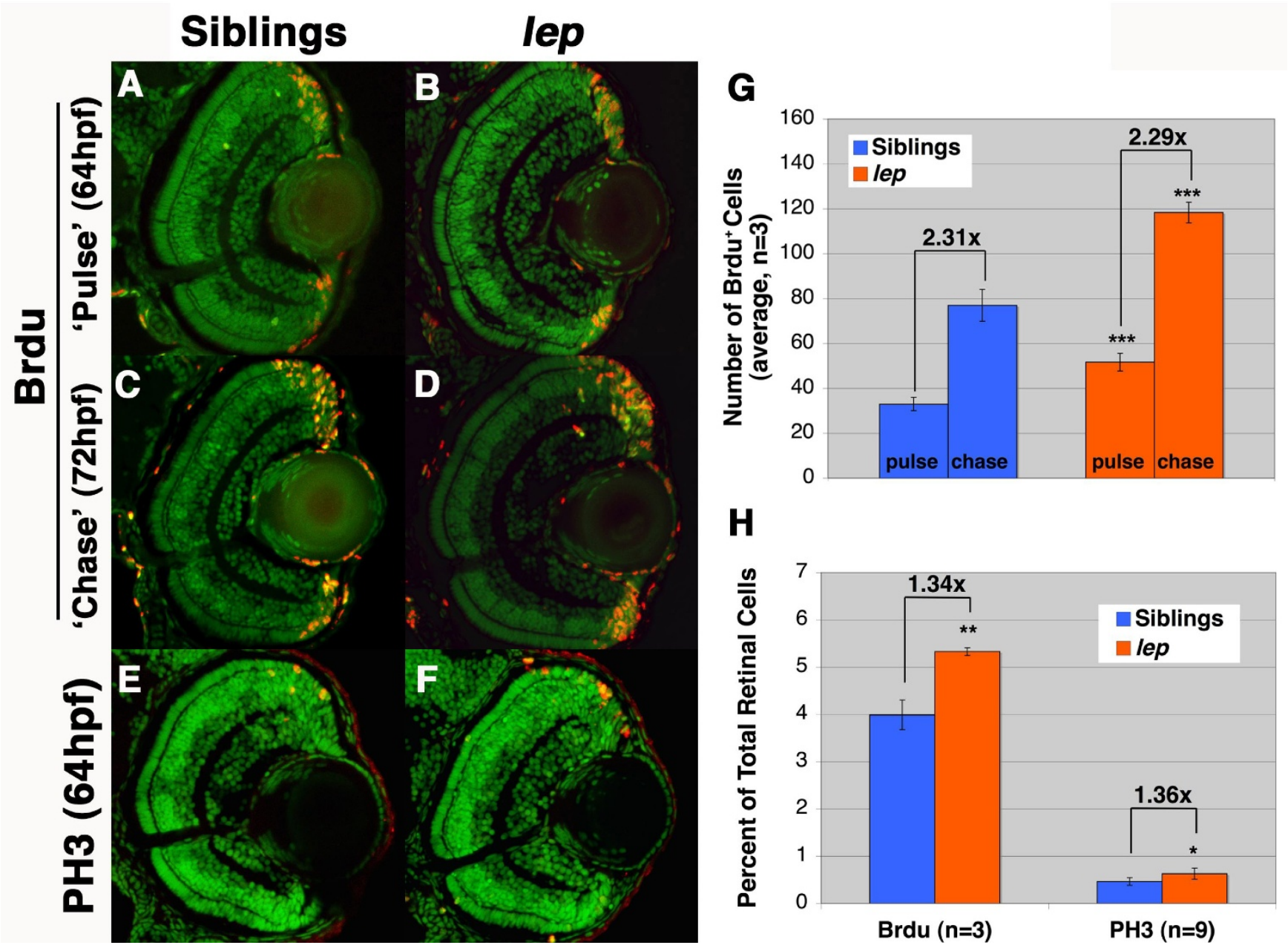

Figure 7

The number of proliferating $C M Z$ progenitors is increased in lep/ptc2, while proliferation rate is unaffected. (A, B) BrdU 'pulse' in sibling (A) and lep/ptc2 mutants (B). BrdU' cells are observed throughout the CMZ. (C, D) After an 8 hour 'chase', BrdU' cells are observed at the CMZ, as well as within the retina of sibling (C) and lep/ptc2 mutants (D). E, F) PH3 labeling in sibling (E) and lep/ptc2 (F) at 64hpf. G) Quantification of BrdU' cells in sibling and lep/ptc2 after 'pulse-chase' experiment. lep/ptc2 retinas contained $67 \%$ more BrdU+ cells than sibling retinas at $64 \mathrm{hpf}$. After an 8 hour 'chase', the number of BrdU' cells increased by almost identical ratios in siblings ( $2.3 \mathrm{I}$-fold increase) and lep/ptc2 mutants (2.29-fold increase, $* * * \mathrm{p}<0.000 \mathrm{I})$. $\mathrm{H}$ ) Quantification of BrdU- and PH3-positive cells as a percentage of total retinal cells in lep/ptc2 and wild-type siblings at 64hpf. The proportion of both $\mathrm{BrdU}^{+}$and $\mathrm{PH}^{+}$cells increased by similar ratios (I.34-fold increase, ${ }^{* *} \mathrm{p}<0.00 \mathrm{I}$ and I.36-fold increase, $*_{p}<0.01$, respectively).

arises from an expansion of the progenitor cell population therein, and not from changes in the length of the cell cycle in these progenitor cells.

\section{Discussion}

Retinal patterning is normal in lep/ptc2 mutants

Characterization of lep/ptc2 retinas with respect to cell type composition revealed that over-activity of the $\mathrm{Hh}$ pathway did not affect retinal patterning. Cell count analysis of all major retinal neuronal cell types revealed no change in the proportion of each cell type. This finding was surprising in light of the well established role of the
Hh pathway in patterning and cell fate decisions throughout the CNS in a number of model systems $[20,38,39]$, as well as studies in Xenopus which show that the overexpression of Shh results in early cell cycle exit [9], and that can, in turn, influence cell fate decisions [8]. While mouse Ptc1+/- (the orthologue of the zebrafish ptc2 [33]) mutants were found to possess no defects in retinal cell fate specification, $\mathrm{Ptc1} \%$ mice could not be examined since they die in utero [37]. Our results indicate that Ptc2dependent Hh signaling does not likely play an integral role in neuronal cell fate decisions in the zebrafish retina. These findings raise the possibility that the second 
zebrafish Patched protein (Patched1) might compensate for the lack of a functional Patched2. While patched1 (blowout) mutant retinas contain all major retinal cell types, the lack of a retinal phenotype might be due to the hypomorphic nature of the mutation $[40,41]$. However, this seems unlikely since patched 1 transcripts are not expressed at detectable levels in retinal tissue throughout development (data not shown and [35]). The reduced number of differentiated Müller glia, a late born cell type [10], could feasibly be due to a bias towards early born cell types in lep/ptc2 mutants; however, this potential bias does not affect neuronal composition of the retina at $5 \mathrm{dpf}$. It is possible that a transient bias towards early neuronal cell types could occur early in retinal development, but any differences could masked by neurons added from the CMZ later in development. Conversely, any bias could also be present in neuronal populations that arise at the $\mathrm{CMZ}$, resulting in an unnoticeable change in neuronal composition of the $5 \mathrm{dpf} l e p / p t c 2$ retina. With this in mind, it will be interesting to examine the proportion of retinal cell types in juvenile lep/ptc2 mutants, as some homozygous fish reach two months of age [18]. Finally, the Hh pathway could play a role in neuronal cell fate decisions in the retina through Hh ligand interactions with other Hh receptors. The Ihog Hh receptors have recently been suggested to compete with Patched for Hh binding [42] and act at levels at, or upstream of, Patched [43]. Indeed, the vertebrate Ihog homologue Cdo is expressed in the developing mouse retina [44]. In the future, it would be interesting to test whether certain aspects of retinal development, such as cell fate decisions, might be Ihog-dependent.

\section{lep/ptc2 mutants as a possible model for the study of BCNS-related ocular pathologies}

In BCNS patients, abnormalities at the interface between the retina and the vitreous, known as epiretinal membranes (ERMs), are associated with disruptions of the ILM and are thought to result from the overproliferation and ectopic presence of multiple cell types, including glia [45]. BCNS ocular pathologies have been linked to mutations in the human PTCH gene $[34,46,47]$, and have been shown to be associated with reactive Müller glia [34]. Müller glial reactivity is often marked by upregulation of GFAP, and lep/ptc2 mutants possessed regions of elevated GFAP levels. Elevated GFAP levels were consistently localized in the inner retina, where Müller glial endfeet terminate and contribute to the ILM. Staining with GS, which labels the Müller glial endfeet, revealed disruptions in ILM integrity in $\sim 40 \%$ of mutant retinas assayed. In these cases, Müller glial endfeet did not terminate properly in the ILM, and the ILM was discontinuous. In some human patients, ERMs are found adjacent to retinal arteries [34]; interestingly, we consistently detected the presence of reactive glial endfeet in localized retinal regions adjacent to the optic nerve and embryonic retinal vasculature. Importantly, no increases in cell death were detected in the retina or optic nerve of lep/ptc2 mutants, or in Müller glia themselves (see Additional file 1), ruling out reactivity due to apoptosis. Finally, in other contexts, Müller glia reactivity is often coupled with ectopic proliferation of these cells during 'reactive gliosis' [48]. BrdU analysis did not detect ectopic proliferation in the central retinas of lep/ ptc2 mutants (Figure 7 and Additional file 1), indicating that while a subset of these cells was reactive, they did not undergo a proliferative response. While reactive Müller glia have been shown to be associated with ERMs, it is unclear whether reactive Müller glia are the cause of these pathologies or, conversely, whether Müller glia become reactive in response to ERM formation. Further studies will be required to answer this question and to shed light on the cellular and molecular causes of ERM formation.

\section{The retinal progenitor population of the $C M Z$ is expanded in lep/ptc2 mutants}

Exact roles for Hh pathway activity in proliferating retinal progenitor cells remain unclear. Over-expression studies in Xenopus suggest a direct role for the Hh pathway in regulating proliferation by influencing the length of the cell cycle in CMZ progenitors [9]. In Ptc1+/- mice, more retinal progenitors are allocated to the $\mathrm{CMZ}$ throughout development and into adulthood [37]. BrdU pulse-chase analysis of the progenitor population at the lep/ptc2 CMZ suggests that Patched-dependent $\mathrm{Hh}$ signaling controls the number of retinal progenitors in the zebrafish $\mathrm{CMZ}$, and does not directly affect the length of the cell cycle in these cells. Importantly, cell counts at an earlier time-point, before the formation of the $\mathrm{CMZ}$, revealed no increase in cell number in lep/ptc2 retinas as compared to their wildtype siblings (Figure 2E), suggesting that the increase in proliferating progenitors at the $\mathrm{CMZ}$ is not simply due to an earlier proliferative event. Shh has been shown to control stem cell maintenance in multiple organs, including the adult brain (reviewed in [49]). Indeed, the Hh pathway genes smo, gli2, and gli3 are expressed in the putative stem cell region of the Xenopus CMZ [14]. In light of our findings, it is possible that the increase in retinal progenitors in the lep/ptc2 CMZ is an indirect result of mis-regulation of the stem cell population rather than a direct effect on progenitor proliferation.

\section{Conclusion}

The possible role for the Hh pathway in retinal cell fate decisions has yet to be established in vivo. Our results indicate that Patched2-dependent Hh signaling is not likely to play an integral role in dictating neuronal cell fate decisions in the zebrafish retina. In addition, ocular phenotypes in lep/ptc2 mutants that are similar to those found in human BCNS patients point to the utility of the lep/ptc2 mutant line as a model for the study of BCNS ocular 
pathologies. With regards to progenitor proliferation, our data support a role for Patched2-dependent Hh signaling in the control of the size of progenitor populations at the retinal $\mathrm{CMZ}$, and not in regulating their rate of proliferation, during zebrafish eye development.

\section{Methods \\ Zebrafish maintenance and strains}

Zebrafish (Danio rerio) were maintained at $28.5^{\circ} \mathrm{C}$ on a 14 $\mathrm{h}$ light/10 h dark cycle. Embryos were obtained from the natural spawning of heterozygous carriers setup in pairwise crosses. Embryos were collected and raised at $28.5^{\circ} \mathrm{C}$ [50] and were staged according to [51]. ptc $2^{\text {tj222 }}$ outcrosses were obtained from the Zebrafish International Resource Center and were propagated by repeated outcrosses to $A B$ fish. All animals were treated in accordance with provisions established at the University of Texas at Austin governing animal use and care.

\section{Histology}

Histology was performed as described in [52]. Briefly, mutant and wild-type sibling embryos were collected and fixed overnight at $4{ }^{\circ} \mathrm{C}$ in a solution of $1 \%(\mathrm{w} / \mathrm{v})$ paraformaldehyde (PFA), 2.5\% glytaraldehyde and 3\% sucrose in phosphate in a $2 \% \mathrm{OsO}_{4}$ solution, washed $3 \times 5 \mathrm{~min}$ in PBS at room temperature and further dehydrated $2 \times 10$ min in propylene oxide and infiltrated $1-2 \mathrm{~h}$ in a $50 \%$ propylene oxide/50\% Epon/Araldite mixture (Polysciences, Inc.). Embryos were then incubated overnight at RT in $100 \%$ Epon/Araldite resin with caps open to allow for propylene oxide evaporation and resin infiltration, embedded and baked at $60^{\circ} \mathrm{C}$ for 2-3 days. Sections 1$1.25 \mu \mathrm{m}$ were cut, mounted on glass slides and stained in a $1 \%$ methylene blue/1\% borax solution. Sections were mounted in DPX (Electron Microscopy Sciences) and photographed on a Leica DMRB microscope mounted with a DFC320 digital camera. For $5 \mathrm{dpf}$ cell counts, the number nuclei in each retinal layer were counted in ten eye sections obtained from different embryos. Horizontal cells were identified by location and morphology. Averages were analyzed and compared using a Student's t-test.

\section{Immunohistochemistry}

Immunohistochemistry was performed as described in [53]. Briefly, embryos were collected and fixed overnight at $4{ }^{\circ} \mathrm{C}$ in a $4 \%$ PFA solution in PBS. Embryos were then washed $3 \times 10 \mathrm{~min}$ in PBS and incubated in a $25 \%$ sucrose/PBS solution for $1 \mathrm{~h}$ followed by $35 \%$ sucrose/ PBS for $1 \mathrm{~h}$. Embryos were then mounted in Tissue Freezing Medium (Triangle Biomedical Sciences, Inc.) and immediately transferred to $-80^{\circ} \mathrm{C}$ freezer for storage. Frozen blocks were sectioned at $12 \mu \mathrm{m}$, mounted on gelatin coated slides, and let dry for $2 \mathrm{~h}$. Slides were then rehydrated in PBTD [0.1\% Tween, 1\% DMSO in $1 \times$ PBS] and then blocked using 5\%NGS/PBTD for $2 \mathrm{~h}$. Slides were incubated in primary antibody diluted in block in a humid chamber over-night at $4^{\circ} \mathrm{C}$. When necessary, nuclear staining was obtained by applying Sytox-Green (Molecular Probes) diluted 1:10,000 in block immediately after removal of primary antibody. Slides were washed $3 \times 10$ min with PBTD and then applied with secondary antibody in block for $1 \mathrm{~h}$. After $3 \times 10 \mathrm{~min}$ washes in PBTD, slides were mounted with Vectashield (Vector Laboratories, Inc.) and coverslipped. Samples were stored at $4{ }^{\circ} \mathrm{C}$ for up to one week and imaged on a Zeiss LSM5 Pascal laser scanning confocal microscope. The following primary antibodies were used: bipolar cells (PKC, 1:200, Santa Cruz Biotechnology), Müller glia (GFAP-zrf1, 1:100, ZIRC and GS, 1:500, Millipore), rods (zpr3, 1:100, ZIRC), green/red cones (zpr1, 1:100, ZIRC), PH3 (1:200, Upstate). Blue opsin (1:500) and UV opsin (1:1,000) antibodies were provided by David Hyde (Univerity of Notre Dame). Amacrine cell antibody (5E11, 1:100) was the gift of Jim Fadool. For cell counts, positively stained cells and nuclei were counted in four eye sections obtained from different embryos. For total cell counts at 33hpf, SytoxGreen staining was used. Averages were analyzed and compared using a Student's t-test.

\section{BrdU incorporation assays}

Embryos were dechorionated and incubated in fish water with $10 \mu \mathrm{M}$ 5-Bromo-2-deoxyuridine (BrdU, Sigma) and $15 \%$ DMSO at $4{ }^{\circ} \mathrm{C}$ for mentioned time periods and either immediately sacrificed or washed three times in fish water and grown at $28.5^{\circ} \mathrm{C}$ prior to sacrifice after [54]. Embryos were processed for immunohistochemistry as in [40]. Mouse anti-BrdU was used at a 1:50 dilution and Cy3 anti-mouse secondary at a 1:200 dilution. Nuclei were counterstained with Sytox:Green (1:10,000, Molecular Probes). For cell counts, Brdu-positive cells and nuclei were counted in four eye sections obtained from different embryos. Averages were analyzed and compared using a Student's t-test.

\section{In-situ hybridization}

Hybridizations were performed as described in [55] using digoxigenin labeled antisense RNA probes. ptc2 probe synthesis construct was provided by Brian Perkins (Texas A\&M University).

\section{Authors' contributions}

JB carried out all experimental procedures. JB and JMG conceived of the study, participated in its design and coordination, and drafted the manuscript. All authors read and approved the final manuscript. 


\section{Additional material}

\section{Additional file 1}

lep/ptc2 mutants do not possess ectopic cell death or proliferation in the central retina and/or optic stalk. (A, B) BrdU incorporation and (C, D) TUNEL assays in $5 \mathrm{dpf}(\mathrm{A}, \mathrm{C})$ phenotypically wild-type siblings and $(B, D)$ lep/ptc2 mutants.

Click here for file

[http://www.biomedcentral.com/content/supplementary/1471213X-9-52-S1.TIFF]

\section{Acknowledgements}

This work was funded by NIH ROI-EYI8005 to J.M.G. We are grateful to David Hyde, Jim Fadool and Brian Perkins for providing reagents, to lan Mackaye for technical assistance and to members of the Gross lab for helpful suggestions on this work, and comments on this manuscript. cDNA constructs and antisera were obtained from the Zebrafish International Resource Center, supported by NIH-NCRR grant P40 RROI 2546.

\section{References}

I. Neumann CJ: Hedgehogs as negative regulators of the cell cycle. Cell Cycle 2005, 4: I I 39-40.

2. Marti E, Bovolenta P: Sonic hedgehog in CNS development: one signal, multiple outputs. Trends Neurosci 2002, 25:89-96.

3. Li Z, Hu M, Ochocinska MJ, Joseph NM, Easter SS Jr: Modulation of cell proliferation in the embryonic retina of zebrafish (Danio rerio). Dev Dyn 2000, 21 9:391-40I.

4. Amato MA, Boy S, Perron M: Hedgehog signaling in vertebrate eye development: a growing puzzle. Cell Mol Life Sci 2004, 61:899-910.

5. Neumann CJ, Nuesslein-Volhard C: Patterning of the zebrafish retina by a wave of sonic hedgehog activity. Science 2000 , 289:2137-9.

6. Shkumatava A, Fischer S, Muller F, Strahle U, Neumann C): Sonic hedgehog, secreted by amacrine cells, acts as a short-range signal to direct differentiation and lamination in the zebrafish retina. Development 2004, I 3 I:3849-58.

7. Stenkamp DL, Frey RA: Extraretinal and retinal hedgehog signaling sequentially regulate retinal differentiation in zebrafish. Dev Biol 2003, 258:349-63.

8. Ohnuma S, Hopper S, Wang KC, Philpott A, Harris WA: Co-ordinating retinal histogenesis: early cell cycle exit enhances early cell fate determination in the Xenopus retina. Development 2002, I 29:2435-46.

9. Locker M, Agathocleous M, Amato MA, Parain K, Harris WA, Perron $M$ : Hedgehog signaling and the retina: insights into the mechanisms controlling the proliferative properties of neural precursors. Genes Dev 2006, 20:3036-48.

10. Schmitt EA, Dowling JE: Early retinal development in the zebrafish, Danio rerio: light and electron microscopic analyses. J Comp Neurol 1999, 404:5I5-36.

II. Raymond PA, Barthel LK, Bernardos RL, Perkowski JJ: Molecular characterization of retinal stem cells and their niches in adult zebrafish. BMC Dev Biol 2006, 6:36.

12. Wetts R, Serbedzija GN, Fraser SE: Cell lineage analysis reveals multipotent precursors in the ciliary margin of the frog retina. Dev Biol 1989, 136:254-63.

13. Perron M, Kanekar S, Vetter ML, Harris WA: The genetic sequence of retinal development in the ciliary margin of the Xenopus eye. Dev Biol 1998, 199:185-200.

14. Perron M, Boy S, Amato MA, Viczian A, Koebernick K, Pieler T, Harris WA: A novel function for Hedgehog signalling in retinal pigment epithelium differentiation. Development 2003, 130:1565-77.

15. Moshiri A, McGuire CR, Reh TA: Sonic hedgehog regulates proliferation of the retinal ciliary marginal zone in posthatch chicks. Dev Dyn 2005, 233:66-75.
16. Stenkamp DL, Frey RA, Mallory DE, Shupe EE: Embryonic retinal gene expression in sonic-you mutant zebrafish. Dev Dyn 2002, 225:344-50.

17. Jensen AM, Wallace VA: Expression of Sonic hedgehog and its putative role as a precursor cell mitogen in the developing mouse retina. Development 1997, 124:363-7|.

18. Koudijs MJ, den Broeder MJ, Keijser A, Wienholds E, Houwing S, van Rooijen EM, Geisler R, van Eeden FJ: The zebrafish mutants dre, uki, and lep encode negative regulators of the hedgehog signaling pathway. PLoS Genet 2005, I:el 9.

19. Johnson RL, Milenkovic L, Scott MP: In vivo functions of the patched protein: requirement of the $C$ terminus for target gene inactivation but not Hedgehog sequestration. Mol Cell 2000, 6:467-78.

20. Goodrich LV, Milenkovic L, Higgins KM, Scott MP: Altered neural cell fates and medulloblastoma in mouse patched mutants. Science 1997, 277: I109-13.

21. Murone M, Rosenthal A, de Sauvage FJ: Sonic hedgehog signaling by the patched-smoothened receptor complex. Curr Biol 1999, 9:76-84.

22. Taipale J, Cooper MK, Maiti T, Beachy PA: Patched acts catalytically to suppress the activity of Smoothened. Nature 2002, 418:892-7.

23. Gross JM, Perkins BD, Amsterdam A, Egana A, Darland T, Matsui JI, Sciascia S, Hopkins N, Dowling JE: Identification of zebrafish insertional mutants with defects in visual system development and function. Genetics 2005, I 70:245-6I.

24. Wang YP, Dakubo G, Howley P, Campsall KD, Mazarolle CJ, Shiga SA, Lewis PM, McMahon AP, Wallace VA: Development of normal retinal organization depends on Sonic hedgehog signaling from ganglion cells. Nat Neurosci 2002, 5:83I-2.

25. Dakubo GD, Mazerolle C, Furimsky M, Yu C, St-Jacques B, McMahon $A P$, Wallace VA: Indian hedgehog signaling from endothelial cells is required for sclera and retinal pigment epithelium development in the mouse eye. Dev Biol 2008, 320:242-55.

26. Karlstrom RO, Talbot WS, Schier AF: Comparative synteny cloning of zebrafish you-too: mutations in the Hedgehog target gli2 affect ventral forebrain patterning. Genes Dev 1999, 13:388-93.

27. Swindell EC, Zilinski CA, Hashimoto R, Shah R, Lane ME, Jamrich M: Regulation and function of foxe 3 during early zebrafish development. Genesis 2008, 46:177-83.

28. Heisenberg CP, Brand M, Jiang YJ, Warga RM, Beuchle D, van Eeden FJ, Furutani-Seiki M, Granato M, Haffter P, Hammerschmidt M, et al.: Genes involved in forebrain development in the zebrafish, Danio rerio. Development 1996, 123:19|-203.

29. Zhang XM, Yang XJ: Regulation of retinal ganglion cell production by Sonic hedgehog. Development 200I, I 28:943-57.

30. Wallace VA: Proliferative and cell fate effects of Hedgehog signaling in the vertebrate retina. Brain Res 2008, I I 92:61-75.

31. Vihtelic TS, Doro CJ, Hyde DR: Cloning and characterization of six zebrafish photoreceptor opsin cDNAs and immunolocalization of their corresponding proteins. Vis Neurosci 1999, 16:57|-85.

32. Gabriel R, Wilhelm M, Straznicky C: Morphology and distribution of Muller cells in the retina of the toad Bufo marinus. Cell Tissue Res 1993, 272:183-92.

33. Lewis KE, Concordet JP, Ingham PW: Characterisation of a second patched gene in the zebrafish Danio rerio and the differential response of patched genes to Hedgehog signalling. Dev Biol 1999, 208: 14-29.

34. Black GC, Mazerolle CJ, Wang Y, Campsall KD, Petrin D, Leonard BC, Damji KF, Evans DG, McLeod D, Wallace VA: Abnormalities of the vitreoretinal interface caused by dysregulated Hedgehog signaling during retinal development. Hum Mol Genet 2003, 12:3269-76.

35. Stenkamp DL, Frey RA, Prabhudesai SN, Raymond PA: Function for Hedgehog genes in zebrafish retinal development. Dev Biol 2000, 220:238-52.

36. Soules KA, Link BA: Morphogenesis of the anterior segment in the zebrafish eye. BMC Dev Biol 2005, 5: 12.

37. Moshiri A, Reh TA: Persistent progenitors at the retinal margin of ptc+/- mice. J Neurosci 2004, 24:229-37.

38. Ekker SC, Ungar AR, Greenstein P, von Kessler DP, Porter JA, Moon RT, Beachy PA: Patterning activities of vertebrate hedgehog 
proteins in the developing eye and brain. Curr Biol 1995, 5:944-55.

39. Ericson J, Rashbass P, Schedl A, Brenner-Morton S, Kawakami A, van Heyningen V, Jessell TM, Briscoe J: Pax6 controls progenitor cell identity and neuronal fate in response to graded Shh signaling. Cell 1997, 90:169-80.

40. Lee J, Willer JR, Willer GB, Smith K, Gregg RG, Gross JM: Zebrafish blowout provides genetic evidence for PatchedI-mediated negative regulation of Hedgehog signaling within the proximal optic vesicle of the vertebrate eye. Dev Biol 2008, 319:10-22.

4I. Koudijs MJ, den Broeder MJ, Groot E, van Eeden F): Genetic analysis of the two zebrafish patched homologues identifies novel roles for the hedgehog signaling pathway. BMC Dev Biol 2008, 8: 15 .

42. McLellan JS, Zheng X, Hauk G, Ghirlando R, Beachy PA, Leahy DJ: The mode of Hedgehog binding to Ihog homologues is not conserved across different phyla. Nature 2008, 455:979-83.

43. McLellan JS, Yao S, Zheng X, Geisbrecht BV, Ghirlando R, Beachy PA, Leahy DJ: Structure of a heparin-dependent complex of Hedgehog and Ihog. Proc Natl Acad Sci USA 2006, 103:17208-13.

44. Mulieri PJ, Okada A, Sassoon DA, McConnell SK, Krauss RS: Developmental expression pattern of the cdo gene. Dev Dyn 2000, 21 9:40-9.

45. Harada C, Mitamura $\mathrm{Y}$, Harada T: The role of cytokines and trophic factors in epiretinal membranes: involvement of signal transduction in glial cells. Prog Retin Eye Res 2006, 25: I49-64.

46. Hahn H, Wicking C, Zaphiropoulous PG, Gailani MR, Shanley S, Chidambaram A, Vorechovsky I, Holmberg E, Unden AB, Gillies S, et al.: Mutations of the human homolog of Drosophila patched in the nevoid basal cell carcinoma syndrome. Cell 1996, 85:84I-5I.

47. Johnson RL, Rothman AL, Xie J, Goodrich LV, Bare JW, Bonifas JM, Quinn AG, Myers RM, Cox DR, Epstein EH Jr, et al.: Human homolog of patched, a candidate gene for the basal cell nevus syndrome. Science 1996, 272:1668-7I.

48. Dyer MA, Cepko CL: Control of Muller glial cell proliferation and activation following retinal injury. Nat Neurosci 2000, 3:873-80.

49. Fuccillo M, Joyner AL, Fishell G: Morphogen to mitogen: the multiple roles of hedgehog signalling in vertebrate neural development. Nat Rev Neurosci 2006, 7:772-83.

50. Westerfield M: The zebrafish book. A guide for the laboratory use of zebrafish (Danio rerio). 4th edition. Eugene: University of Oregon Press; 2000.

5I. Kimmel CB, Ballard WW, Kimmel SR, Ullmann B, Schilling TF: Stages of embryonic development of the zebrafish. Dev Dyn 1995, 203:253-310.

52. Nuckels RJ, Gross JM: Histological preparation of embryonic and adult zebrafish eyes. Cold Spring Harbor Protocols 2007:2.

53. Uribe RA, Gross JM: Immunohistochemistry on cryosections from embryonic and adult zebrafish eyes. Cold Spring Harbor Protocols 2007:2.

54. Masai I, Yamaguchi M, Tonou-Fujimori N, Komori A, Okamoto H: The hedgehog-PKA pathway regulates two distinct steps of the differentiation of retinal ganglion cells: the cell-cycle exit of retinoblasts and their neuronal maturation. Development 2005, 132:1539-53.

55. Jowett $T$, Lettice $L:$ Whole-mount in situ hybridizations on zebrafish embryos using a mixture of digoxigenin- and fluorescein-labelled probes. Trends Genet 1994, 10:73-4.
Publish with Biomed Central and every scientist can read your work free of charge

"BioMed Central will be the most significant development for disseminating the results of biomedical research in our lifetime. "

Sir Paul Nurse, Cancer Research UK

Your research papers will be:

- available free of charge to the entire biomedical community

- peer reviewed and published immediately upon acceptance

- cited in PubMed and archived on PubMed Central

- yours - you keep the copyright

Submit your manuscript here:

http://www.biomedcentral.com/info/publishing_adv.asp
BioMedcentral 